\title{
SLOW MAPPINGS OF FINITE DISTORTION
}

\author{
JANI ONNINEN AND PEKKA PANKKA
}

\begin{abstract}
We examine mappings of finite distortion between Riemannian manifolds. We use integral type isoperimetric inequalities to obtain Liouville type growth results under mild assumptions on the distortion of the mappings and the geometry of the manifolds.
\end{abstract}

\section{INTRODUCTION}

According to the classical Euclidean theory of quasiregular mappings bounded entire quasiregular mappings are constant. A mapping $f: \mathbb{R}^{n} \rightarrow \mathbb{R}^{n}$ in the Sobolev space $W_{\text {loc }}^{1, n}\left(\mathbb{R}^{n}, \mathbb{R}^{n}\right)$ is $K$-quasiregular if

$$
\|D f\|^{n} \leqslant K J_{f} \text { a.e., }
$$

where $\|D f\|$ is the operator norm of the tangent map of $f$ and $J_{f}$ is the Jacobian determinant.

This version of the classical Liouville's theorem can be derived from the following qualitative lower growth bound estimate: Given $n \geqslant 2$ and $K \geqslant 1$ there exists a constant $\alpha>0$ depending only on $n$ and $K$ so that every $K$-quasiregular mapping $f: \mathbb{R}^{n} \rightarrow \mathbb{R}^{n}$ satisfying

$$
\lim _{|x| \rightarrow \infty}|x|^{-\alpha}|f(x)|=0
$$

is constant [16, III.1.13].

These two growth results of Liouville type admit far reaching generalizations for quasiregular mappings into closed manifolds. Let $\mathbb{Y}$ be a closed, connected, and oriented Riemannian $n$-manifold receiving a non-constant $K$-quasiregular mapping from $\mathbb{R}^{n}$. On the one hand, Varopoulos's theorem [20, Theorem X.11] states that the fundamental group of $\mathbb{Y}$ is virtually nilpotent and has the growth of order at most $n$. On the other hand, Bonk and Heinonen [1, Theorem 1.11] showed that quasiregular mappings into non-trivial quasiregularly elliptic manifolds have a uniform lower bound in growth rate in terms of averaged counting function. Precisely, their result tells that if $\mathbb{Y}$ has a non-trivial de Rham cohomology ring then

$$
\liminf _{r \rightarrow \infty} \frac{1}{r^{\alpha}} \int_{B^{n}(r)} J_{f}>0
$$

2000 Mathematics Subject Classification. 30C65 (53C21 58A12).

Onninen was supported by the NSF grant DMS-0632409 and Pankka by grants DMS0353549 and DMS-0757732. 
where $\alpha>0$ depends only on $n$ and $K$. Here and in what follows $B^{n}(r)$ stands for the open Euclidean $n$-ball centered at the origin of radius $r$.

In this paper we show that both of these theorems hold for a wider class of mappings termed mappings of finite distortion and we give an interpretation of the theorems through isoperimetric inequalities for mappings. To be more precise, we consider continuous Sobolev mappings $f: \mathbb{R}^{n} \rightarrow \mathbb{Y}$ in $W_{\text {loc }}^{1, n}\left(\mathbb{R}^{n}, \mathbb{Y}\right)$, where $\mathbb{Y}$ is a connected and oriented Riemannian $n$-manifold. A mapping $f \in W_{\text {loc }}^{1, n}\left(\mathbb{R}^{n}, \mathbb{Y}\right)$ has finite distortion provided there exists a measurable function $K: \mathbb{R}^{n} \rightarrow[1, \infty)$ such that

$$
\|D f(x)\|^{n} \leqslant K(x) J_{f}(x) \text { for a.e. } x \in \mathbb{R}^{n} .
$$

This class of mappings is intriguing. Such mappings may be seen as natural generalizations of quasiregular mappings. Indeed, under a certain integrability condition on the distortion function, mappings of finite distortion have geometric and topological behavior similar to quasiregular mappings between Euclidean $n$-domains; see the book by Iwaniec and Martin [9], and the references there.

According to our first theorem here, mappings of $p$-mean distortion, $p>$ $n-1$, exhibit a similar growth rate as quasiregular mappings. This result generalizes the theorem of Bonk and Heinonen. We say that $f$ has mean distortion in $L^{p}, 1 \leqslant p<\infty$, if

$$
\mathcal{K}_{p}=\mathcal{K}_{f, p}=\sup _{r \geqslant 1}\left(f_{B^{n}(r)} K_{f}(x)^{p} \mathrm{~d} x\right)^{1 / p}<\infty .
$$

Theorem 1. Let $\mathbb{Y}$ be a closed, connected, and oriented Riemannian $n$ manifold, $n \geqslant 2$, and $f: \mathbb{R}^{n} \rightarrow \mathbb{Y}$ a non-constant slow mapping of finite distortion with mean distortion in $L^{n-1}$. Then $\operatorname{dim} H^{\ell}(\mathbb{Y})=0$ for $1<\ell<$ $n-1$. If $f$ has mean distortion in $L^{p}$ for some $p>n-1$, then $\mathbb{Y}$ is a rational homology sphere.

We say that a mapping of finite distortion $f: \mathbb{R}^{n} \rightarrow \mathbb{Y}$ is slow if

$$
\lim _{r \rightarrow \infty} \frac{1}{r^{\alpha}} \int_{B^{n}(r)} J_{f}=0
$$

for every $\alpha>0$.

Our second theorem gives an interpretation of Varopoulos's theorem as an end point in a spectrum of growth results.

Theorem 2. Let $\mathbb{Y}$ be an open and oriented Riemannian $n$-manifold, $n \geqslant 2$, supporting a d-dimensional isoperimetric inequality, $d \geqslant 2$, and $f: \mathbb{R}^{n} \rightarrow \mathbb{Y}$ a mapping of finite distortion with mean distortion in $L^{n-1}$. Then $f$ is

(a) constant if $d>n$,

(b) either constant or $f$ has at least logarithmic growth if $d<n$, and

(c) either constant or $f$ is not slow if $d=n$. 
A mapping of finite distortion $f: \mathbb{R}^{n} \rightarrow \mathbb{Y}$ has at least logarithmic growth if

$$
\liminf _{r \rightarrow \infty} \frac{1}{(\log r)^{\alpha}} \int_{B^{n}(r)} J_{f}>0
$$

for some $\alpha>0$.

The original proof of Bonk and Heinonen is based on non-linear Hodge theory and $\mathcal{A}$-harmonic potential theory. In contrast, the proof of Varopoulos's theorem is based on an isoperimetric inequality and uses the fact that a closed manifold admitting a non-constant quasiregular mapping from $\mathbb{R}^{n}$ does not have a conformally hyperbolic universal cover. It seems that especially the method of Bonk and Heinonen relies heavily on the uniform boundedness of the distortion function and, therefore, is not available in our case. Our proofs are surprisingly simple, purely analytic, and use no conformal geometry. Indeed, the proofs of Theorems 1 and 2 are based on the existence of integral type isoperimetric inequalities for suitable Sobolev mappings, and an interplay between volume growth and distortion.

The main tool in the proof of Theorem 1 is the concept of Cartan forms [5]. This method has two advantages. First, the geometry of the domain plays only a small role and the necessary geometric properties can easily be axiomatized. Second, the obtained isoperimetric inequalities allow us to assume that the mean distortion of the mapping is in $L^{n-1}$. In the Euclidean theory of mappings of finite distortion, the $L^{n-1}$-integrability assumption on the distortion function is considered to be the minimal requirement for topological conclusions. For instance, the full analogue of Reshetnyak's theorem is conjectured to hold if $K \in L_{\text {loc }}^{n-1}$ and known under a slightly stronger integrable assumption on $K$; that is, a non-constant mapping of finite distortion is both discrete and open provided $K \in L_{\text {loc }}^{p}, p>n-1$ [12]. Apart from continuity, our techniques do not rely on topological properties of the mappings.

In the case of Theorem 2, we obtain an isoperimetric inequality for mappings through Gagliardo-Nirenberg-Sobolev type inequalities for BV functions.

Our approach allows us to formulate Theorems 1 and 2 in the context of mappings of finite distortion between Riemannian manifolds (Theorems 13 and 12). In this setting, the cohomological assumptions on the spaces translate into assumptions on the kernel of the induced mapping on the level of de Rham cohomology. The geometric assumptions on the domain manifold $\mathbb{X}$ tend to get quite technical very quickly. They allow us, however, to include mappings from manifolds with boundary. For instance, Theorem 13 covers the case when the domain is the puncture unit ball $\mathbb{B}_{\circ}^{n} \subset \mathbb{R}^{n}$, generalizing the main theorem in [15]. Indeed, one of the reasons to state our results in this general framework is to cover both cases $\mathbb{X}=\mathbb{R}^{n}$ and $\mathbb{X}=\mathbb{B}_{\circ}^{n}$ simultaneously. Furthermore, as in the results of Zorich [24] on the injectivity of quasiregular mappings, the boundedness of mean $L^{p}$-distortion can be relaxed in both theorems. For instance, the mean distortion integral 
in (1.3) can have logarithmic growth at infinity, see Condition (7.13) and Theorems 12 and 13. The sharpness of Condition (7.13) is demonstrated in Example 14.

Acknowledgments. This study stems from a question posed by Tadeusz Iwaniec during Pankka's visit to Syracuse University in spring 2007. Both authors wish to thank Iwaniec for his inspiration and encouragement. Pankka also wishes to thank the Department of Mathematics at Syracuse University for its generous hospitality.

\section{Preliminaries}

Throughout the article we consider continuous Sobolev mappings of the class $W_{\text {loc }}^{1, n}(\mathbb{X}, \mathbb{Y})$, where $\mathbb{X}$ and $\mathbb{Y}$ are connected and oriented Riemannian $n$-manifolds. Our manifolds are not assumed to be closed unless otherwise stated and they can have compact boundaries. If $\mathbb{X}$ has a compact boundary $\partial \mathbb{X}$, we assume that the mapping in question is a restriction of a continuous mapping in $W_{\text {loc }}^{1, n}(\tilde{\mathbb{X}}, \mathbb{Y})$ for some ambient open manifold $\tilde{\mathbb{X}}$ containing $\mathbb{X}$ as a submanifold with boundary.

A Sobolev mapping $f \in W_{\text {loc }}^{1, n}(\mathbb{X}, \mathbb{Y})$ induces a pull-back homomorphism $f^{*}: C^{\infty}\left(\bigwedge^{\ell} \mathbb{Y}\right) \rightarrow L_{\text {loc }}^{n / \ell}(\mathbb{X})$ locally of the form

$$
f^{*}\left(u d x_{i_{1}} \wedge \cdots \wedge d x_{i_{\ell}}\right)=(u \circ f) d\left(x_{i_{1}} \circ f\right) \wedge \cdots \wedge d\left(x_{i_{\ell}} \circ f\right) .
$$

Moreover, the pull-back $f^{*}$ commutes with the exterior derivative, that is, $d \circ f^{*}=f^{*} \circ d$, where the left hand side is understood in the weak sense. By the pointwise inequality, $\left|f^{*} \alpha\right| \leqslant|D f|^{\ell}(|\alpha| \circ f)$ for $\alpha \in C^{\infty}\left(\bigwedge^{\ell} \mathbb{Y}\right)$, we can conclude that $f^{*} \alpha \in W_{\text {loc }}^{d, n / \ell}\left(\bigwedge^{\ell} \mathbb{X}\right)$ for all closed forms $\alpha \in C^{\infty}\left(\bigwedge^{\ell} \mathbb{X}\right)$. Here $W_{\text {loc }}^{d, p}\left(\bigwedge^{\ell} \mathbb{X}\right)$ is the local partial Sobolev space of $\ell$-forms, see [10] for more details.

The pull-back homomorphism $f^{*}$ induced by a mapping $f$ induces in turn a natural homomorphism $f^{*}: H^{\ell}(\mathbb{Y}) \rightarrow H^{\ell}(\mathbb{X})$, where $H^{\ell}(\mathbb{X})$ and $H^{\ell}(\mathbb{Y})$ are the $\ell$ th-de Rham cohomology groups of $\mathbb{X}$ and $\mathbb{Y}$, respectively. A priori, $f^{*}$ induces only a homomorphism from $H^{\ell}(\mathbb{Y})$ to the Sobolev-de Rham cohomology $H^{\ell, n / \ell}(\mathbb{X})$ of $\mathbb{X}$, but cohomologies $H^{\ell, n / \ell}(\mathbb{X})$ and $H^{\ell}(\mathbb{X})$ are naturally isomorphic, see e.g. [21, Chapter 5] or [15].

\section{CARTAN FORMS}

In this section we discuss Cartan forms on closed oriented Riemannian manifolds. For a more detailed exposition we refer to [5].

We say that a smooth $n$-form $\omega$ on a closed Riemannian $n$-manifold $\mathbb{Y}$ is $a$ Cartan form if there exist closed forms $\xi \in C^{\infty}\left(\bigwedge^{\ell} \mathbb{Y}\right)$ and $\zeta \in C^{\infty}\left(\bigwedge^{n-\ell} \mathbb{Y}\right)$ for some $\ell \in\{1, \ldots, n-1\}$ so that

$$
\omega-\xi \wedge \zeta
$$


is exact. Note that by [5, Proposition 2.3] our definition is equivalent with the one given in [5].

According to Proposition 2.4 in [5] every $n$-form $\omega$ on $\mathbb{Y}$ is a Cartan form if and only if $H^{\ell}(\mathbb{Y}) \neq 0$ for some $\ell \in\{1, \ldots, n-1\}$. In particular, if $\mathbb{Y}$ is cohomologically non-trivial, the volume form $\operatorname{vol}_{g}$ of a Riemannian metric $g$ of $\mathbb{Y}$ is a Cartan form. Moreover, for every non-trivial cohomology class $c \in H^{\ell}(\mathbb{Y})$ the volume form $\operatorname{vol}_{g}$ can be represented using the harmonic $\ell$-form $\xi \in C^{\infty}\left(\bigwedge^{\ell} \mathbb{Y}\right)$ in $c$ as

$$
\operatorname{vol}_{g}=\left(\frac{1}{\sqrt{\lambda}} \xi\right) \wedge *\left(\frac{1}{\sqrt{\lambda}} \xi\right)+d \tau
$$

for some $(n-1)$-form $\tau$, where

$$
\lambda=\int_{\mathbb{Y}} \xi \wedge * \xi>0,
$$

see the proof of Proposition 2.4 in [5]. If $\lambda=1$, we say that the harmonic form $\xi$ almost splits the volume form $\operatorname{vol}_{g}$. If, in addition, $d \tau=0$ in (3.5), we say that $\xi$ splits $\operatorname{vol}_{g}$. Product manifolds with product metrics give examples of manifolds with splitting volume forms.

To measure the defect in splitting of the volume form of metric $g$, we denote for every harmonic $\ell$-form $\xi$ almost splitting vol $_{g}$ the splitting defect of $\xi$ by

$$
C_{g}(\xi)=\inf _{\tau}\left(\|\xi\|_{\infty}^{2}+\|\tau\|_{\infty}\right)
$$

where the infimum is taken over all $(n-1)$-forms $\tau$ satisfying (3.5). Furthermore, we say that the maximal splitting defect of $g$ is

$$
C_{g}=\sup _{\xi} C_{g}(\xi)
$$

where the supremum is taken over all non-trivial harmonic forms. The maximal splitting defect of $g$ is finite by finite dimensionality of $H^{*}(\mathbb{Y})$.

\section{Controlled exhaustions}

In this section we define a class of exhaustions of Riemannian manifolds. Our exhaustions are modeled after Mattila and Rickman [13] and Zorich [24]. Having these exhaustions at our disposal, we define mappings of $p$-mean distortion. It is worth keeping in mind two important examples, $\mathbb{R}^{n}$ with the exhaustion $\left\{\mathbb{B}^{n}(r): r \in[0, \infty)\right\}$ and the closed exterior ball $\mathbb{R}^{n} \backslash \mathbb{B}^{n}$ with an exhaustion $\left\{\mathbb{B}^{n}(r) \backslash \mathbb{B}^{n}: r>1\right\}$. The exterior ball $\mathbb{R}^{n} \backslash \mathbb{B}^{n}$ equipped with this exhaustion gives a conformally equivalent but a metrically complete model for a punctured unit ball $\overline{\mathbb{B}}_{\text {o }}^{n}$ with the exhaustion $\left\{\overline{\mathbb{B}}^{n} \backslash \overline{\mathbb{B}}^{n}(r): 0<r<1\right\}$.

Let $\mathbb{X}$ be a complete Riemannian $n$-manifold possibly with a compact boundary $\partial \mathbb{X}$. We say that $\mathcal{D}=\left\{D_{t}: t \in I\right\}$, where the index set $I \subset[0, \infty)$ is measurable, is an exhaustion of $\mathbb{X}$ if

$$
\left(D_{1}\right) \mathbb{X}=\bigcup_{t \in I} D_{t},
$$


$\left(D_{2}\right) \overline{D_{s}} \subset D_{t}$ for $s<t$,

$\left(D_{3}\right) D_{t}$ is a relatively compact open set and $\partial D_{t}$ is a closed $(n-1)$ submanifold of $\mathbb{X}$ for all $t \in I$, and

$\left(D_{4}\right) \partial \mathbb{X}$ is a boundary component of $D_{t}$ for every $t \in I$.

We say that an exhaustion $\mathcal{D}$ is asymptotically small if there exist positive constants $t_{\mathcal{D}}, c_{D}, c_{I}$, and $c_{A}$ so that

$\left(D_{5}\right)|I \cap(0, t)| \geqslant c_{D} t$ for $t \in I \cap\left(t_{\mathcal{D}}, \infty\right)$,

$\left(D_{6}\right)$

$$
\frac{\mathrm{d}}{\mathrm{d} t} \int_{D_{t}} u \geqslant c_{I} \int_{\partial D_{t}} u
$$

for almost every $t \in I \cap\left(t_{\mathcal{D}}, \infty\right)$ for every non-negative $u \in L_{\text {loc }}^{1}(\mathbb{X})$, and

$\left(D_{7}\right)\left|\partial D_{t}\right| \leqslant c_{A} t^{n-1}$ for $t \in I \cap\left(t_{\mathcal{D}}, \infty\right)$ and for some $c_{A} \geqslant 1$.

Finally, we impose the following two conditions controlling the geometry of differential forms with respect to the exhaustion. An exhaustion $\mathcal{D}$ is said to admit small extendable potentials if for every $t \in I$

$\left(D_{8}\right)$ the inclusion $\iota: \partial D_{t} \rightarrow \overline{D_{t}}$ induces a surjection on $\iota^{*}: H^{\ell}\left(D_{t}\right) \rightarrow$ $H^{\ell}\left(\partial D_{t}\right)$ for $1 \leqslant \ell \leqslant n-2$, and

$\left(D_{9}\right)$ for every $p \in(1, n-1)$ there exists $C_{P}=C_{P}(n, p)>0$ such that for every $\omega \in W^{d, p}\left(\bigwedge^{\ell} \partial D_{t}\right)$ there exists a closed form $\omega_{t} \in$ $W^{d, p}\left(\bigwedge^{\ell} \partial D_{t}\right)$ so that

$$
\left(\int_{\partial D_{t}}\left|\omega-\omega_{t}\right|^{p^{*}} \mathrm{~d} \mathcal{H}^{n-1}\right)^{1 / p^{*}} \leqslant C_{P}\left(\int_{\partial D_{t}}|d \omega|^{p} \mathrm{~d} \mathcal{H}^{n-1}\right)^{1 / p},
$$

where $p^{*}=(n-1) p /((n-1)-p)$.

We say that an exhaustion $\mathcal{D}$ satisfying $\left(D_{1}\right)-\left(D_{9}\right)$ is controlled. Furthermore, we refer to constants $c_{\mathcal{D}}, t_{D}, c_{I}, c_{A}, C_{P}$, and set $I$ as the data $[\mathcal{D}]$ of $\mathcal{D}$.

A mapping $f: \mathbb{X} \rightarrow \mathbb{Y}$ has mean distortion in $L^{p}$ with respect to $\mathcal{D}=$ $\left\{D_{t}: t \in I\right\}$ if there exists $t_{0} \geqslant 0$ so that

$$
\hat{K}_{p}=\hat{K}_{f, p}=\sup _{t \in I \cap\left(t_{0}, \infty\right)}\left(f_{\partial D_{t}} K_{f}^{p}\right)^{1 / p}<\infty .
$$

Remark 3. If $\mathbb{X}=\mathbb{R}^{n}$, then for every $R>1$ there exists a subset $J \subset(1, R)$ so that $|J|>R / 4$ and

$$
\sup _{r \in(1, R)}\left(f_{B^{n}(r)} K_{f}^{p}\right)^{1 / p} \lesssim \sup _{r \in J}\left(f_{\partial B^{n}(r)} K_{f}^{p}\right)^{1 / p} \lesssim \sup _{r \in(1, R)}\left(f_{B^{n}(r)} K_{f}^{p}\right)^{1 / p},
$$

where the constants of comparability depend only on $n$ and $p$. Thus, $\mathcal{K}_{p} \sim$ $\hat{K}_{p}$; that is, (1.3) is equivalent with (4.9). 
In spite the lack of an explicit assumption on the conformal geometry, manifolds admitting asymptotically small exhaustions are conformally parabolic. The converse is also true in the sense of the conformal gauge of the metric. For more detailed discussion on conformal types of Riemannian manifolds we refer to [8].

Proposition 4. Let $\left(\mathbb{X}, g_{\mathbb{X}}\right)$ be an open and oriented Riemannian n-manifold. Then $\left(\mathbb{X}, g_{\mathbb{X}}\right)$ is conformally parabolic if and only if there exists a Riemannian metric $g$ conformally equivalent with $g_{\mathbb{X}}$ and an asymptotically small exhaustion $\mathcal{D}$ on $(\mathbb{X}, g)$.

Proof. Since every conformally parabolic manifold $\left(\mathbb{X}, g_{\mathbb{X}}\right)$ admits a complete metric $g$ with finite volume in the conformal gauge of $g_{\mathbb{X}}$, we have by Sard's theorem and coarea formula that the family $\left\{B_{g}\left(x_{0}, r\right): r>0\right\}$ of concentric balls on $\mathbb{X}$ contains an asymptotically small exhaustion $\mathcal{D}$ as a subset.

Suppose now that $(\mathbb{X}, g)$ is a Riemannian manifold with an asymptotically small exhaustion $\mathcal{D}=\left\{D_{t}: t \in I\right\}$. To show that $(\mathbb{X}, g)$ is conformally parabolic, it is sufficient to show that the family of paths leaving every compact set in $\mathbb{X}$ has zero conformal modulus. By the reciprocity of modulus of path families and modulus of separating surfaces families [22], it is sufficient to show that the surface family $\Sigma=\left\{\partial D_{t}: t \in I\right\}$ has infinite conformal modulus. Let $\rho$ be an admissible function on $\Sigma$ and let

$$
\varphi(t)=\int_{D_{t}} \rho^{\frac{n}{n-1}}
$$

for $t \in I$. Then $\varphi: I \rightarrow[0, \infty]$ is non-decreasing.

By $\left(D_{5}\right)$, we may fix $q>2 / c_{D}$ such that $\left|I \cap\left(q^{j-1}, q^{j}\right)\right|>q^{j-1}$ for $j \geqslant 1$. By $\left(D_{6}\right),\left(D_{7}\right)$, and Hölder's inequality,

$$
\begin{aligned}
\varphi\left(q^{j}\right)-\varphi\left(q^{j-1}\right) & \geqslant \int_{I \cap\left(q^{j-1}, q^{j}\right)} \varphi^{\prime}(t) \mathrm{d} t \gtrsim \int_{I \cap\left(q^{j-1}, q^{j}\right)}\left(\int_{\partial D_{t}} \rho^{\frac{n}{n-1}}\right) \mathrm{d} t \\
& \gtrsim \int_{I \cap\left(q^{j-1}, q^{j}\right)}\left|\partial D_{t}\right|^{-\frac{1}{n-1}}\left(\int_{\partial D_{t}} \rho\right)^{\frac{n}{n-1}} \mathrm{~d} t \\
& \gtrsim \int_{q^{j}-q^{j-1}}^{q^{j}} \frac{1}{t}=\log \left(\frac{q}{q-1}\right),
\end{aligned}
$$

where constants of comparability depend only on $c_{I}, c_{A}$, and $n$. Thus every $\Sigma_{j}=\left\{\partial D_{t}: t \in I \cap\left(q^{j-1}, q^{j}\right)\right\}$ has conformal modulus uniformly bounded from below. Since families $\Sigma_{j}$ are disjoint, $\Sigma$ has infinite conformal modulus.

Let us now discuss the implications of conditions $\left(D_{8}\right)$ and $\left(D_{9}\right)$. Condition $\left(D_{9}\right)$ imposes restrictions to the geometry of the differential forms on $\mathbb{X}$. Indeed, $\left(D_{9}\right)$ requires the manifold to support a uniform Sobolev-Poincaré inequality on submanifolds $\partial D_{t}$. Let us recall that every closed manifold supports a Poincaré inequality for differential forms with a constant depending on the geometry of the manifold [10, Theorem 6.4]. Together with 
the Sobolev embedding theorem this yields the Sobolev-Poincaré inequality (4.8) for every manifold $\partial D_{t}$ with a constant depending on the geometry of the manifold $\partial D_{t}$ and $p$. The role of $\left(D_{9}\right)$ is therefore only to impose an additional condition that this constant is independent on the submanifold $\partial D_{t}$.

For the reader's convenience we give a sketch of a proof of the SobolevPoincaré inequality for differential forms on closed manifolds. For the notation, we refer to $[19$, Section 2].

Lemma 5. Let $\mathbb{Y}$ be a closed oriented Riemannian $n$-manifold and $p \in$ $(1, n)$. Then there exists a constant $C_{P}=C_{P}(p, \mathbb{Y})>0$ with the following property: For every $\omega \in W^{d, p}\left(\bigwedge^{\ell} \mathbb{Y}\right)$ there exists a closed form $\omega_{0} \in L^{p}\left(\bigwedge^{\ell} \mathbb{Y}\right)$ such that $\omega-\omega_{0} \in W^{1, p}\left(\bigwedge^{\ell} \mathbb{Y}\right)$ and

$$
\left(\int_{\mathbb{Y}}\left|\omega-\omega_{0}\right|^{p^{*}}\right)^{1 / p^{*}} \leqslant C_{P}\left(\int_{\mathbb{Y}}|d \omega|^{p}\right)^{1 / p},
$$

where $p^{*}=\frac{n p}{n-p}$.

Sketch of a proof. Let $\omega \in W^{d, p}\left(\bigwedge^{\ell} \mathbb{Y}\right)$. By [10, Theorem 6.4], there exists $C>0$, depending only on $\mathbb{Y}$ and $p$, and $\omega_{0} \in L^{p}\left(\bigwedge^{\ell} \mathbb{Y}\right)$ such that $\omega-\omega_{0} \in$ $W^{1, p}\left(\bigwedge^{\ell} \mathbb{Y}\right)$ and

$$
\left\|\omega-\omega_{0}\right\|_{W^{1, p}\left(\bigwedge^{\ell} \mathbb{Y}\right)} \leqslant C\|d \omega\|_{p}
$$

Since $\omega-\omega_{0} \in W^{1, p}\left(\bigwedge^{\ell} \mathbb{Y}\right)$, we have that $\left|\omega-\omega_{0}\right| \in W^{1, p}(\mathbb{Y})$. Thus, by the Sobolev embedding theorem on manifolds, see e.g. [6, Theorem 3.5],

$$
\left\|\omega-\omega_{0}\right\|_{p^{*}} \leqslant C^{\prime}\left\|\nabla\left|\omega-\omega_{0}\right|\right\|_{p} .
$$

By the Gaffney type inequality of Scott [19, Proposition 4.5],

$$
\left\|\nabla\left(\omega-\omega_{0}\right)\right\|_{p}^{p} \leqslant C^{\prime \prime}\left(\left\|d\left(\omega-\omega_{0}\right)\right\|_{p}^{p}+\left\|d^{*}\left(\omega-\omega_{0}\right)\right\|_{p}^{p}\right) .
$$

Thus the claim follows from

$$
\left\|\nabla\left|\omega-\omega_{0}\right|\right\|_{p} \leqslant\left\|\nabla\left(\omega-\omega_{0}\right)\right\|_{p}
$$

as in the proof of [10, Theorem 6.4].

In contrast to the other requirements on the exhaustion, $\left(D_{8}\right)$ imposes restrictions to the topology of $\mathbb{X}$ in scales controlled by the exhaustion. Having $\left(D_{8}\right)$ at our disposal, the inclusion mappings $\partial D_{t} \hookrightarrow \bar{D}_{t}$ induce surjections $W^{d, p}\left(\bigwedge^{\ell} D_{t}\right) \rightarrow W^{d, p}\left(\bigwedge^{\ell} \partial D_{t}\right)$ for every $t \in I$.

We end this section with two examples on manifolds and their controlled exhaustions.

Example 6. (1) The basic examples of controlled exhaustions are exhaustion of $\mathbb{R}^{n}$ with balls $\mathbb{B}^{n}(r)$ and $\mathbb{R}^{n} \backslash \mathbb{B}^{n}$ with annuli $\mathbb{B}^{n}(r) \backslash \mathbb{B}^{n}$. Indeed, in these cases it suffices to verify conditions $\left(D_{8}\right)$ and $\left(D_{9}\right)$. Whereas $\left(D_{8}\right)$ follows from cohomological triviality of $S^{n-1},\left(D_{9}\right)$ follows from similarity of boundary components of exhausting sets. 
(2) The previous example can be further modified to allow topologically non-trivial domains. Let

$$
\mathbb{X}=\cdots \# \mathbb{X}_{k} \# \mathbb{X}_{k+1} \# \cdots
$$

where $\mathbb{X}_{k}$ are closed, connected, and oriented Riemannian n-manifolds. If manifolds $\mathbb{X}_{k}$ and $\mathbb{X}_{k+1}$ are glued in a geometrically controlled fashion, $\mathbb{X}$ admits a controlled exhaustion. To be more precise, suppose that there exists $\delta>0$ such that the neighborhood of the gluing is isometric to $S^{n-1}(r) \times$ $(-\delta, \delta)$ for some $r>0$. In this case, we may take such an exhaustion $\left\{D_{t}: t \in I\right\}$ that components of $\partial D_{t}$ are similar to $S^{n-1}$.

\section{ISOPERIMETRIC INEQUALITY FOR SOBOLEV MAPPINGS}

In this section, we show that an isoperimetric inequality of the target space yields an integral type isoperimetric inequality for continuous Sobolev mappings in $W^{1, n}$.

We say that $\mathbb{Y}$ supports a $d$-dimensional isoperimetric inequality with a constant $C_{\mathbb{Y}}>0$ if if $\mathbb{Y}$ satisfies $(1, \varphi)$-isoperimetric inequality with $C_{\mathbb{Y}}>0$, that is,

$$
\varphi(|\Omega|)|\Omega| \leqslant C_{\mathbb{Y}}|\partial \Omega|
$$

for all domains $\Omega \subset \mathbb{Y}$, where

$$
\varphi(r)=\min \left\{r^{-1 / d}, r^{-1 / n}\right\}= \begin{cases}r^{-1 / d}, & r \geqslant 1 \\ r^{-1 / n}, & r<1 .\end{cases}
$$

Theorem 7. Let $n \geqslant 2$ and $d \geqslant 2$. Let $\mathbb{X}$ be a complete, open, and oriented Riemannian $n$-manifold and $\mathcal{D}=\left\{D_{t}: t \in I\right\}$ an exhaustion of $\mathbb{X}$. Suppose that $\mathbb{Y}$ is a connected and oriented Riemannian n-manifold supporting a $d$-dimensional isoperimetric inequality with a constant $C_{\mathbb{Y}}>0$. Let also $f: \mathbb{X} \rightarrow \mathbb{Y}$ be a continuous Sobolev mapping in $W_{\mathrm{loc}}^{1, n}(\mathbb{X}, \mathbb{Y})$. Then there exists $C=C\left(C_{\mathbb{Y}}, d\right)>0$ so that for almost every $t \in I$ we have

(i)

$$
\int_{D_{t}} J_{f} \leqslant C\left(\int_{\partial D_{t}}\left|D^{\#} f\right|\right)^{\frac{d}{d-1}} \quad \text { if } d \geqslant n, \text { and }
$$

$$
\int_{D_{t}} J_{f} \leqslant C \max \left\{\left(\int_{D_{t}} J_{f}\right)^{1 / n},\left(\int_{D_{t}} J_{f}\right)^{1 / d}\right\}\left(\int_{\partial D_{t}}\left|D^{\#} f\right|\right) \quad \text { if } d<n \text {. }
$$

Although the following lemma is certainly well-known, we include a proof for the reader's convenience.

Lemma 8. Let $\mathbb{X}$ be an open and oriented Riemannian $n$-manifold, $\Omega \subset \mathbb{X}$ an open set with a smooth boundary, $\mathbb{Y}$ an oriented Riemannian $n$-manifold, and $f: \mathbb{X} \rightarrow \mathbb{Y}$ a smooth mapping. Then

$$
y \mapsto \operatorname{deg}(y, \Omega ; f)
$$


is a $B V$ function on $\mathbb{Y}$ and

$$
|D \operatorname{deg}(\cdot, \Omega ; f)|(\mathbb{Y}) \leqslant \int_{\partial \Omega}\left|D^{\#} f\right| .
$$

An $L^{1}$-function $u: \mathbb{Y} \rightarrow \mathbb{R}$ is a said to be $B V$ if

$$
|D u|(\mathbb{Y}):=\sup \left\{\int_{\mathbb{Y}} u \operatorname{div} \varphi \operatorname{vol}_{\mathbb{Y}}: \varphi \in C_{0}^{\infty}(T \mathbb{Y}),|\varphi| \leqslant 1\right\}<\infty .
$$

Proof of Lemma 8. Let $\varphi$ be a compactly supported smooth vector field on $\mathbb{Y}$. Then, by definition,

$$
\operatorname{div} \varphi \operatorname{vol}_{\mathbb{Y}}=d(\varphi\llcorner\operatorname{vol} \mathbb{Y})
$$

and by Stokes' theorem,

$$
\int_{\Omega} d f^{*}\left(\varphi\left\llcorner\operatorname{vol}_{\mathbb{Y}}\right)=\int_{\partial \Omega} \iota^{*} f^{*}\left(\varphi\left\llcorner\operatorname{vol}_{\mathbb{Y}}\right) .\right.\right.
$$

Thus

$$
\begin{aligned}
\int_{\Omega}(\operatorname{div} \varphi) \circ f J_{f} \operatorname{vol}_{\mathbb{X}} & =\int_{\Omega} f^{*}\left(\operatorname{div} \varphi \operatorname{vol}_{\mathbb{Y}}\right)=\int_{\Omega} f^{*}\left(d\left(\varphi\left\llcorner\operatorname{vol}_{\mathbb{Y}}\right)\right)\right. \\
& =\int_{\Omega} d f^{*}\left(\varphi\left\llcorner\operatorname{vol}_{\mathbb{Y}}\right)=\int_{\partial \Omega} \iota^{*} f^{*}\left(\varphi\left\llcorner\operatorname{vol}_{\mathbb{Y}}\right)\right.\right. \\
& \leqslant \int_{\partial \Omega} \mid D^{\#} f \| \varphi\left\llcorner\operatorname{vol}_{\mathbb{Y}}\left|\circ f \leqslant\|\varphi\|_{\infty} \int_{\partial \Omega}\right| D^{\#} f \mid .\right.
\end{aligned}
$$

By the change of variables,

$$
\begin{aligned}
\left|\int_{\mathbb{Y}}(\operatorname{div} \varphi) \operatorname{deg}(\cdot, \Omega ; f) \operatorname{vol}_{\mathbb{Y}}\right| & =\left|\int_{\Omega}(\operatorname{div} \varphi) \circ f J_{f} \operatorname{vol}_{\mathbb{X}}\right| \\
& \leqslant\|\varphi\|_{\infty} \int_{\partial \Omega}\left|D^{\#} f\right| .
\end{aligned}
$$

This concludes the proof.

The following $L^{1}$ estimate for BV functions is a combination of results of Coulhon, Grigorýan, and Levin [2, Prop 2.1] and Miranda, Pallara, Paronetto, and Preunkert [14, Prop 1.4].

Lemma 9. Let $\mathbb{Y}$ support a d-dimensional isoperimetric inequality, $2 \leqslant d \leqslant$ $n$, with constant $C_{\mathbb{Y}}>0$ and let $u: \mathbb{Y} \rightarrow \mathbb{Z}$ be a compactly supported $B V$ function on $\mathbb{Y}$. Then

$$
\|u\|_{1} \leqslant C \max \left\{\|u\|_{1}^{1 / d},\|u\|_{1}^{1 / n}\right\}|D u|(\mathbb{Y}),
$$

where $C=C\left(C_{\mathbb{Y}}\right)>0$

Proof. By considering $\tilde{\varphi}=\varphi / C_{\mathbb{Y}}$ if necessary, we may assume that $C_{\mathbb{Y}}=1$. Since $\mathbb{Y}$ supports $d$-dimensional isoperimetric inequality, by [2, Prop. 2.1] it also supports F-Sobolev inequality for compactly supported Lipschitz functions, that is,

$$
\int_{\mathbb{Y}}|v| F\left(\frac{v}{\|v\|_{1}}\right) \leqslant \int_{\mathbb{Y}}|\nabla v|
$$


where $F(r)=c \varphi(2 / r)$, for every $v \in \operatorname{Lip}_{0}(\mathbb{Y})$. Here $c>0$ is universal.

Suppose now that $u \in B V(\mathbb{Y})$ is compactly supported and let $\Omega$ be a relatively compact domain of $\mathbb{Y}$ containing the support of $u$. By [14, Prop. 1.4], there exists a sequence $\left(u_{k}\right)$ in $C_{0}^{\infty}(\Omega)$ so that $u_{k} \rightarrow u$ in $L^{1}$ and

$$
|D u|(\mathbb{Y})=\lim _{k \rightarrow \infty} \int_{\mathbb{Y}}\left|\nabla u_{k}\right| .
$$

Thus, by continuity of $F$ and Fatou's lemma, we have

$$
\begin{aligned}
\int_{\mathbb{Y}}|u| F\left(\frac{|u|}{\|u\|_{1}}\right) & \leqslant \liminf _{k \rightarrow \infty} \int_{\mathbb{Y}}\left|u_{k}\right| F\left(\frac{\left|u_{k}\right|}{\left\|u_{k}\right\|_{1}}\right) \\
& \leqslant \liminf _{k \rightarrow \infty} \int_{\Omega}\left|\nabla u_{k}\right|=|D u|(\mathbb{Y}) .
\end{aligned}
$$

Let $\Omega^{\prime}=\left\{y:|u(y)| \leqslant 2\|u\|_{1}\right\}$. Then

$$
F\left(\frac{|u(y)|}{\|u\|_{1}}\right)=c \varphi\left(2 \frac{\|u\|_{1}}{|u(y)|}\right)= \begin{cases}c\left(\frac{u(y)}{2\|u\|_{1}}\right)^{1 / n}, & y \notin \Omega^{\prime} \\ c\left(\frac{u(y)}{2\|u\|_{1}}\right)^{1 / d}, & y \in \Omega^{\prime}\end{cases}
$$

Thus

$$
\begin{aligned}
\|u\|_{1} & =\int_{\mathbb{Y}}|u|=\int_{\Omega^{\prime}}|u|+\int_{\mathbb{Y} \backslash \Omega^{\prime}}|u| \\
& \leqslant \int_{\Omega^{\prime}}|u|^{1+\frac{1}{d}}+\int_{\mathbb{Y} \backslash \Omega^{\prime}}|u|^{1+\frac{1}{n}} \\
& \leqslant C\left(\int_{\Omega^{\prime}}|u| F\left(\frac{|u|}{\|u\|_{1}}\right)\|u\|_{1}^{1 / d}+\int_{\mathbb{Y} \backslash \Omega^{\prime}}|u| F\left(\frac{|u|}{\|u\|_{1}}\right)\|u\|_{1}^{1 / n}\right) \\
& \leqslant C \max \left\{\|u\|_{1}^{1 / d},\|u\|_{1}^{1 / n}\right\} \int_{\mathbb{Y}}|u| F\left(\frac{|u|}{\|u\|_{1}}\right) \\
& \leqslant C \max \left\{\|u\|_{1}^{1 / d},\|u\|_{1}^{1 / n}\right\}|D u|(\mathbb{Y}),
\end{aligned}
$$

where $C>0$ is universal.

Proof of Theorem \%. Let $\left(f_{k}\right)$ be a sequence of smooth mappings $\mathbb{X} \rightarrow \mathbb{Y}$ tending to $f$ in $W_{\text {loc }}^{1, n}(\mathbb{X}, \mathbb{Y})$, see [17] and [18].

Suppose first that $d \geqslant n$. Since $r^{\frac{d-1}{d}} \leqslant r^{\frac{n-1}{n}}$ for $r \leqslant 1$, by the $d$ dimensional isoperimetric inequality there exists $C=C\left(C_{\mathbb{Y}}\right)>0$ so that

$$
|\Omega| \leqslant C|\partial \Omega|^{\frac{d}{d-1}}
$$

for all domains $\Omega$ in $\mathbb{Y}$. Thus $\mathbb{Y}$ supports a classical Gagliardo-NirenbergSobolev inequality

$$
\|u\|_{\frac{d}{d-1}} \leqslant|D u|(\mathbb{Y})
$$


for BV functions $u$ on $\mathbb{Y}$, see e.g. [7, 3.30] and [3, 5.6.2]. Thus, by Lemma 8 , we have

$$
\left\|\operatorname{deg}\left(\cdot, D_{t}, f_{k}\right)\right\|_{\frac{d}{d-1}} \leqslant C_{\mathbb{Y}}\left|D \operatorname{deg}\left(\cdot, D_{t}, f_{k}\right)\right|(\mathbb{Y}) \leqslant C_{\mathbb{Y}} \int_{\partial D_{t}}\left|D^{\#} f_{k}\right|
$$

for every $t \in I$ and every $k$. Since $\operatorname{deg}\left(\cdot, D_{t}, f_{k}\right)$ is integer valued,

$$
\int_{D_{t}} J_{f_{k}}=\int_{\mathbb{Y}} \operatorname{deg}\left(\cdot, D_{t}, f_{k}\right) \leqslant \int_{\mathbb{Y}} \mid \operatorname{deg}\left(\cdot, D_{t}, f_{k}\right)^{\frac{d}{d-1}} \leqslant\left(C_{\mathbb{Y}} \int_{\partial D_{t}}\left|D^{\#} f_{k}\right|\right)^{\frac{d}{d-1}}
$$

for every $t \in I$.

Suppose now that $\mathbb{Y}$ supports a $d$-dimensional isoperimetric inequality for $2 \leqslant d<n$ with a constant $C_{\mathbb{Y}}$. By Lemma 9 and Lemma 8 together with the change of variables, we obtain

$$
\int_{D_{t}} J_{f_{k}} \leqslant C \max \left\{\left(\int_{D_{t}} J_{f_{k}}\right)^{1 / n},\left(\int_{D_{t}} J_{f_{k}}\right)^{1 / d}\right\}\left(\int_{\partial D_{t}}\left|D f_{k}\right|^{n-1}\right) .
$$

for every $t \in I$ and every $k$. Thus for every $d \geqslant 2$, the claim holds for smooth mappings $f_{k}$.

By a usual telescoping decomposition of the Jacobian in local coordinates $[9,8.1]$, we obtain

as $k \rightarrow \infty$.

$$
\int_{D_{t}} J_{f_{k}} \rightarrow \int_{D_{t}} J_{f}
$$

By $\left(D_{6}\right)$ and using the telescoping decomposition again, we have

$$
\begin{aligned}
\int_{I \cap(0, s)} \int_{\partial D_{t}}|| D^{\#} f_{k}|-| D^{\#} f|| \mathrm{d} t & \leqslant \frac{1}{c_{I}} \int_{D_{s}}|| D^{\#} f_{k}|-| D^{\#} f|| \\
& \leqslant \frac{1}{c_{I}} \int_{D_{s}}\left|D^{\#} f_{k}-D^{\#} f\right| \rightarrow 0
\end{aligned}
$$

for every $s>t_{\mathcal{D}}$ as $k \rightarrow \infty$. Thus

$$
\int_{\partial D_{t}}\left|D^{\#} f_{k}\right| \rightarrow \int_{\partial D_{t}}\left|D^{\#} f\right|
$$

for almost every $t \in I$. The claim follows.

\section{Cohomological isoperimetric inequality for Sobolev MAPPINGS}

In this section we study mappings into closed target manifolds. We prove that the non-trivial kernel of the induced pull-back mapping yields an integral type isoperimetric inequality for mappings. This extends the result of Giannetti and Passarelli di Napoli [4].

Theorem 10. Let $\mathbb{X}$ be a connected, open, and oriented Riemannian $n$ manifold, $n \geqslant 2$, and $\mathcal{D}=\left\{D_{t}: t \in I\right\}$ a controlled exhaustion of $\mathbb{X},\left(\mathbb{Y}, g_{\mathbb{Y}}\right)$ a closed, connected, and oriented Riemannian n-manifold, and $f: \mathbb{X} \rightarrow \mathbb{Y}$ a continuous Sobolev mapping in $W_{\mathrm{loc}}^{1, n}(\mathbb{X}, \mathbb{Y})$. Let $p \in[n-1, n)$. If either 
(i) $p>n-1$ and $\operatorname{ker} f^{*} \neq 0$, or

(ii) $p=n-1, n \geqslant 4$, and there exists $\ell \in\{2, \ldots, n-2\}$ such that $\operatorname{ker}\left(f^{*}: H^{\ell}(\mathbb{Y}) \rightarrow H^{\ell}(\mathbb{X})\right) \neq 0$,

then

$$
\int_{D_{t}} J_{f} \leqslant C\left|\partial D_{t}\right|^{\frac{n}{n-1}-\frac{n}{p}}\left(\int_{\partial D_{t}}|D f|^{p}\right)^{\frac{n}{p}}+C \int_{\partial D_{t}}|D f|^{n-1}
$$

for almost every $t \in I$, where $C=C\left(n, p, C_{P}, C_{g_{\mathbb{Y}}}\right)$.

For the proof we recall the following version of Stokes' theorem.

Lemma 11. Let $\mathbb{X}$ be an open and oriented Riemannian manifold, $\left\{D_{t}: t \in\right.$ $I\}$ an exhaustion satisfying $\left(D_{6}\right)$, and $\omega \in W_{\mathrm{loc}}^{d, p}\left(\bigwedge^{n-1} \mathbb{X}\right), p>1$. Then, for almost every $t \in I, \iota^{*} \omega \in W^{d, p}\left(\bigwedge^{n-1} \partial D_{t}\right)$ and

$$
\int_{\partial D_{t}} \iota^{*} \omega=\int_{D_{t}} d \omega
$$

Furthermore, if $t \in I, \omega \in W^{d, p}\left(\bigwedge^{n-1} \bar{D}_{t}\right)$ is weakly closed, and $\iota^{*} \omega \in$ $W^{d, p}\left(\bigwedge^{n-1} \partial D_{t}\right)$ then

$$
\int_{\partial D_{t}} \iota^{*} \omega=0
$$

Proof. By the density of smooth forms, we may fix a sequence $\left(\omega_{k}\right)$ such that $\omega_{k} \rightarrow \omega$ in $W_{\mathrm{loc}}^{d, p}\left(\bigwedge^{\ell} \mathbb{X}\right)$. Since

$$
\int_{I \cap(0, s)} \int_{\partial D_{t}}\left|\iota^{*}\left(\omega-\omega_{k}\right)\right|^{p} \mathrm{~d} t \leqslant \frac{1}{c_{I}} \int_{D_{s}}\left|\iota^{*}\left(\omega-\omega_{k}\right)\right|^{p} \leqslant \frac{1}{c_{I}} \int_{D_{s}}\left|\omega-\omega_{k}\right|^{p} \rightarrow 0
$$

as $k \rightarrow \infty$ by $\left(D_{6}\right)$, we have that

$$
\int_{\partial D_{t}}\left|\iota^{*}\left(\omega-\omega_{k}\right)\right|^{p} \rightarrow 0
$$

for almost every $t \in I$. Hence, for almost every $t \in I$,

$$
\int_{D_{t}} d \omega=\lim _{k \rightarrow \infty} \int_{D_{t}} d \omega_{k}=\lim _{k \rightarrow \infty} \int_{\partial D_{t}} \iota^{*} \omega_{k}=\int_{\partial D_{t}} \iota^{*} \omega .
$$

Suppose now that $t \in I, \omega \in W^{d, p}\left(\bigwedge^{n-1} \bar{D}_{t}\right)$ is weakly closed, and $\iota^{*} \omega \in$ $W^{d, p}\left(\bigwedge^{n-1} \partial D_{t}\right)$. Then $\iota^{*} \omega$ is weakly closed and there exist sequences $\left(\eta_{k}\right)$ and $\left(\eta_{k}^{\prime}\right)$ of smooth closed forms in $C^{\infty}\left(\bigwedge^{n-1} \bar{D}_{t}\right)$ and $C^{\infty}\left(\bigwedge^{n-1} U\right)$, where $U$ is a tubular neighborhood of $\partial D_{t}$, such that $\eta_{k} \rightarrow \omega$ and $\iota^{*} \eta_{k}^{\prime} \rightarrow \iota^{*} \omega_{k}$ in $W^{d, p}\left(\bigwedge^{n-1} \bar{D}_{t}\right)$ and $W^{d, p}\left(\bigwedge^{n-1} \partial D_{t}\right)$, respectively. We may assume that $\eta_{k}^{\prime}-\eta_{k}=d \tau_{k}$ in $U \cap D_{t}$ for some smooth $(n-2)$-forms $\tau_{k}$ defined in $U$. Thus we may set $\omega_{k}=\eta_{k}+d\left(\varphi \tau_{k}\right)$, where $\varphi \in C_{0}^{\infty}(U)$ satisfies $\varphi \equiv 1$ in a neighborhood of $\partial D_{t}$. Thus $\iota^{*} \omega_{k}=\iota^{*} \eta_{k}^{\prime}$ and $d \omega_{k}=d \eta_{k}$ in $D_{t}$. Then

$$
\int_{\partial D_{t}} \iota^{*} \omega=\lim _{k \rightarrow \infty} \int_{\partial D_{t}} \iota^{*} \omega_{k}=\lim _{k \rightarrow \infty} \int_{D_{t}} d \omega_{k}=0
$$

The proof is complete. 
Proof of Theorem 10. Under either the assumption $(i)$ or $(i i)$, we may fix a harmonic $\ell$-form $\xi$ on $\mathbb{Y}$ such that $f^{*} \xi$ is weakly exact. Furthermore, we may assume that

$$
\operatorname{vol}_{\mathbb{Y}}=\xi \wedge * \xi+d \tau
$$

where $\tau \in C^{\infty}\left(\bigwedge^{n-1} \mathbb{Y}\right)$. Thus

$$
\int_{D_{t}} J_{f} \operatorname{vol}_{\mathbb{X}}=\int_{D_{t}} f^{*}\left(\operatorname{vol}_{\mathbb{Y}}\right)=\int_{D_{t}} f^{*}(\xi) \wedge f^{*}(* \xi)+\int_{D_{t}} f^{*}(d \tau)
$$

for every $t \in I$. Since

$$
\int_{D_{t}} f^{*}(d \tau)=\int_{D_{t}} d f^{*} \tau=\int_{\partial D_{t}} \iota^{*} f^{*} \tau \leqslant\|\tau\|_{\infty} \int_{\partial D_{t}}|D f|^{n-1}
$$

for almost every $t \in I$ by Stokes' theorem (Lemma 11), it suffices to show that

$$
\int_{D_{t}} f^{*}(\xi) \wedge f^{*}(* \xi) \leqslant C\|\xi\|_{\infty}^{2}\left|\partial D_{t}\right|^{\frac{n}{n-1}-\frac{n}{p}}\left(\int_{\partial D_{t}}|D f|^{p}\right)^{\frac{n}{p}}
$$

for almost every $t \in I$.

Suppose first that $(i)$ holds. Since $f^{*}(\xi)$ is weakly exact, we may fix $\omega \in W_{\text {loc }}^{d, n / \ell}\left(\bigwedge^{\ell-1} \mathbb{X}\right)$ so that $d \omega=f^{*}(\xi)$. Thus, by weak exactness of $f^{*}(* \xi)$,

$$
\int_{D_{t}} f^{*}(\xi) \wedge f^{*}(* \xi)=\int_{D_{t}} d \omega \wedge f^{*}(* \xi)=\int_{D_{t}} d\left(\omega \wedge f^{*}(* \xi)\right)
$$

for every $t \in I$.

We set

$$
q=\frac{p}{\ell}, q^{*}=\frac{(n-1) q}{(n-1)-q}, \text { and } s=\frac{q^{*}}{q^{*}-1} .
$$

Since $\mathcal{D}$ is controlled, for almost every $t \in I$ we may fix a weakly closed form $\tilde{\omega} \in W^{d, q}\left(\bigwedge^{\ell} D_{t}\right)$ such that $\iota^{*} \tilde{\omega} \in W^{d, q^{*}}\left(\bigwedge^{\ell-1} \partial D_{t}\right)$ and

$$
\left(\int_{\partial D_{t}}\left|\iota^{*} \omega-\iota^{*} \tilde{\omega}\right|^{q^{*}} \mathrm{~d} \mathcal{H}^{n-1}\right)^{1 / q^{*}} \leqslant C_{P}\left(\int_{\partial D_{t}}\left|d \iota^{*} \omega\right|^{q} \mathrm{~d} \mathcal{H}^{n-1}\right)^{1 / q} .
$$

Let $\hat{\omega}=\omega-\tilde{\omega}$. Since $\tilde{\omega}$ is closed, we have, by Stokes' theorem,

$$
\int_{\partial D_{t}} \iota^{*} \tilde{\omega} \wedge \iota^{*} f^{*}(* \xi)=\int_{D_{t}} d \tilde{\omega} \wedge f^{*}(* \xi)=0
$$

Thus

$$
\begin{aligned}
\int_{D_{t}} f^{*}(\xi) \wedge f^{*}(* \xi) & =\int_{\partial D_{t}} \iota^{*}\left(\omega \wedge f^{*}(* \xi)\right)=\int_{\partial D_{t}} \iota^{*} \hat{\omega} \wedge \iota^{*} f^{*}(* \xi) \\
& \leqslant C \int_{\partial D_{t}}\left|\iota^{*} \hat{\omega}\right|\left|\iota^{*} f^{*}(* \xi)\right| \leqslant C \int_{\partial D_{t}}\left|\iota^{*} \hat{\omega}\right|\left|f^{*}(* \xi)\right|,
\end{aligned}
$$

where $C$ depends only on $n$. Since

$$
\left|f^{*}(\xi)\right|^{q} \leqslant|D f|^{q \ell}\left(|\xi|^{q} \circ f\right) \leqslant\|\xi\|_{\infty}^{q}|D f|^{p}
$$

and

$$
\left|f^{*}(* \xi)\right|^{s} \leqslant|D f|^{s(n-\ell)}\|* \xi\|_{\infty}^{s}=\|\xi\|_{\infty}^{s}|D f|^{s(n-\ell)}
$$


where

$$
s(n-\ell)=p \frac{(n-1)(n-\ell)}{n p-n \ell+\ell}<p \frac{(n-1)(n-\ell)}{n(n-1)-n \ell+\ell}=p,
$$

we have, by Hölder's inequality and (6.12), that

$$
\begin{aligned}
\int_{\partial D_{t}}\left|\iota^{*} \hat{\omega}\right|\left|f^{*}(* \xi)\right| & \leqslant\left(\int_{\partial D_{t}}\left|\iota^{*} \hat{\omega}\right|^{q^{*}}\right)^{1 / q^{*}}\left(\int_{\partial D_{t}}\left|f^{*}(* \xi)\right|^{s}\right)^{1 / s} \\
& \leqslant C_{P}\left(\int_{\partial D_{t}}|d \hat{\omega}|^{q}\right)^{1 / q}\left(\int_{\partial D_{t}}\left|f^{*}(* \xi)\right|^{s}\right)^{1 / s} \\
& \leqslant C_{P}\left(\int_{\partial D_{t}}\left|f^{*}(\xi)\right|^{q}\right)^{1 / q}\left(\int_{\partial D_{t}}\left|f^{*}(* \xi)\right|^{s}\right)^{1 / s} \\
& \leqslant C_{P}\left|\partial D_{t}\right|^{\frac{n}{n-1}-\frac{n}{p}}\|\xi\|_{\infty}^{2}\left(\int_{\partial D_{t}}|D f|^{p}\right)^{\frac{n}{p}} .
\end{aligned}
$$

This proves (6.11).

Let us now assume $(i i)$. Set

$$
q_{0}=\frac{n-1}{\ell}, q_{0}^{*}=\frac{(n-1) q_{0}}{(n-1)-q_{0}}, \text { and } s_{0}=\frac{q_{0}^{*}}{q_{0}^{*}-1} .
$$

Then $s_{0}(n-\ell)=n-1$. Following the proof above by almost verbatim, we have

$$
\begin{aligned}
\int_{D_{t}} f^{*}(\xi) \wedge f^{*}(* \xi) & \leqslant C_{P}\left(\int_{\partial D_{t}}\left|f^{*}(\xi)\right|^{q_{0}}\right)^{1 / q_{0}}\left(\int_{\partial D_{t}}\left|f^{*}(* \xi)\right|^{s_{0}}\right)^{1 / s_{0}} \\
& \leqslant C_{P}\|\xi\|_{\infty}^{2}\left(\int_{\partial D_{t}}|D f|^{n-1}\right)^{\frac{n}{n-1}}
\end{aligned}
$$

This concludes the proof.

\section{GROWTH OF MAPPINGS OF FINITE DISTORTION}

In this section we state and prove geometric versions of Theorems 1 and 2 . First, we give a sharp geometric version of Theorem 2 in terms of a growth condition of the Jacobian. For this, let $\mathbb{X}$ be a Riemannian $n$-manifold with an exhaustion $\mathcal{D}=\left\{D_{t}: t \in I\right\}, K: \mathbb{X} \rightarrow[1, \infty)$ a measurable function, and $p \geqslant 1$. We set

$$
\mathfrak{K}_{p}(t)=\mathfrak{K}_{K, p}(t)=\sup _{s \in I \cap(0, t]}\left(f_{\partial D_{s}} K^{p}\right)^{1 / p}
$$

and

$$
\psi_{p}(t)=\psi_{\mathfrak{K}_{p}}(t)=\int_{t_{\mathcal{D}}}^{t} \frac{\mathrm{d} s}{s \mathfrak{K}_{p}(s)}
$$

for $t>t_{\mathcal{D}}$. 
Theorem 12. Let $\mathbb{X}$ be a complete, open, and oriented Riemannian $n$ manifold and $\mathcal{D}=\left\{D_{t}: t \in I\right\}$ an asymptotically small exhaustion of $\mathbb{X}$. Suppose that $\mathbb{Y}$ is a connected and oriented Riemannian $n$-manifold supporting a d-dimensional isoperimetric inequality, $d \geqslant 2$, with a constant $C_{\mathbb{Y}}>0$. Let $f: \mathbb{X} \rightarrow \mathbb{Y}$ be a mapping of finite distortion with distortion $K: \mathbb{X} \rightarrow \mathbb{R}$. Suppose that $\sup _{t \in I} \psi_{\mathfrak{K}_{n-1}}(t)=\infty$. Then $f$ is constant if $d>n$.

Furthermore, if $d \leqslant n$, then either $f$ is constant or we have the following two cases:

(a) For $d<n$ there exists $\alpha=\alpha(n, d)>0$ such that

$$
\liminf _{t \rightarrow \infty, t \in I} \frac{1}{\left(\psi_{n-1}\left(c_{D} t\right)\right)^{\alpha}} \int_{D_{t}} J_{f}>0
$$

(b) For $d=n$ there exists $\beta=\beta\left(n, C_{\mathbb{Y}},[\mathcal{D}]\right)>0$ such that

$$
\liminf _{t \rightarrow \infty, t \in I} \frac{1}{e^{\beta \psi_{n-1}\left(c_{D} t\right)}} \int_{D_{t}} J_{f}>0 .
$$

Proof. Suppose first that $d \geqslant n$. By Theorem 7 and Hölder's inequality,

$$
\begin{aligned}
\int_{D_{t}} J_{f} & \leqslant\left(C_{\mathbb{Y}} \int_{\partial D_{t}}\left|D^{\#} f\right|\right)^{\frac{d}{d-1}} \\
& \leqslant C_{\mathbb{Y}}^{\frac{d}{d-1}}\left(\int_{\partial D_{t}} K^{n-1}\right)^{\frac{1}{n} \frac{d}{d-1}}\left(\int_{\partial D_{t}} J_{f}\right)^{\frac{n-1}{n} \frac{d}{d-1}} \\
& \leqslant C_{\mathbb{Y}}^{\frac{d}{d-1}}\left|\partial D_{t}\right|^{\frac{1}{\gamma(n-1)}} \mathfrak{K}_{n-1}(t)^{1 / \gamma}\left(\int_{\partial D_{t}} J_{f}\right)^{1 / \gamma}
\end{aligned}
$$

for almost every $t \in I$, where

$$
\gamma=\frac{n}{n-1} \frac{d-1}{d}
$$

Set

$$
\varphi(t)=\int_{D_{t}} J_{f}
$$

for $t \in I$. Then, by $\left(D_{6}\right)$ and $\left(D_{7}\right)$,

$$
\varphi(t)^{\gamma} \leqslant C_{\mathbb{Y}}^{\frac{n}{n-1}}\left|\partial D_{t}\right|^{\frac{1}{n-1}} \mathfrak{K}_{n-1}(t) \frac{1}{c_{I}} \varphi^{\prime}(t) \leqslant \frac{C_{\mathbb{Y}}^{\frac{n}{n-1}} c_{A}}{c_{I}} t \mathfrak{K}_{n-1}(t) \varphi^{\prime}(t)
$$

for almost every $t \in I$.

Suppose that $f$ is non-constant. Since $f$ is a mapping of finite distortion, there exists $t_{0} \in I$ so that $\varphi\left(t_{0}\right)>0$. 
By $\left(D_{5}\right)$ we have, for every $t>t_{0}$, that

$$
\begin{aligned}
\int_{I \cap\left(t_{0}, t\right)} \frac{\varphi^{\prime}(s)}{\varphi(s)^{\gamma}} \mathrm{d} s & \geqslant \beta \int_{I \cap\left(t_{0}, t\right)} \frac{\mathrm{d} s}{s \mathfrak{K}_{n-1}(s)} \\
& \geqslant \beta \int_{t_{0}}^{t_{0}+\left|I \cap\left(t_{0}, t\right)\right|} \frac{\mathrm{d} s}{s \mathfrak{K}_{n-1}(s)} \\
& =\beta\left(\psi_{n-1}\left(t_{0}+\left|I \cap\left(t_{0}, t\right)\right|\right)-\psi_{n-1}\left(t_{0}\right)\right) \\
& \geqslant \beta\left(\psi_{n-1}\left(c_{D} t\right)-\psi_{n-1}\left(t_{0}\right)\right),
\end{aligned}
$$

where $\beta=\left(\frac{C_{\mathbb{Y}}^{\frac{n}{n-1}} C_{A}}{C_{R}}\right)^{-1}$.

If $d=n$ then $\gamma=1$ and we obtain (b). Indeed, by (7.14),

$$
\varphi(t) \geqslant \varphi\left(t_{0}\right) e^{\beta\left(\psi_{n-1}\left(c_{D} t\right)-\psi_{n-1}\left(t_{0}\right)\right)}
$$

for $t>t_{0}$.

For $d>n$, we have $\gamma>1$ and

$$
\frac{\varphi(t)^{1-\gamma}-\varphi\left(t_{0}\right)^{1-\gamma}}{1-\gamma} \geqslant \beta\left(\psi_{n-1}\left(c_{D} t\right)-\psi_{n-1}\left(t_{0}\right)\right) .
$$

Thus there exists $t>t_{0}$ so that $\varphi(t)<0$. This contradicts the positivity of the Jacobian of $f$. Thus $f$ is constant for $d>n$.

Suppose now that $d<n$. Then, by Theorem 7 ,

$$
\int_{D_{t}} J_{f} \leqslant C\left\{\left(\int_{D_{t}} J_{f}\right)^{1 / n},\left(\int_{D_{t}} J_{f}\right)^{1 / d}\right\}\left(\int_{\partial D_{t}}|D f|^{n-1}\right)
$$

for almost every $t \in I$, where $C=C\left(C_{\mathbb{Y}}, d\right)>0$. We show first that there exists $t_{2}>0$ so that

$$
\int_{D_{t}} J_{f} \leqslant C\left(\int_{\partial D_{t}}|D f|^{n-1}\right)^{\frac{d}{d-1}}
$$

for $t \in I \cap\left(t_{2}, \infty\right)$. It is enough to show that there exists $t \in I$ so that

$$
\varphi(t)=\int_{D_{t}} J_{f}>1
$$

Suppose towards contradiction that $\varphi(t) \leqslant 1$ for all $t \in I$, then (7.15) yields

$$
\int_{D_{t}} J_{f} \leqslant C\left(\int_{\partial D_{t}}|D f|^{n-1}\right)^{\frac{n}{n-1}}
$$

for all $t \in I$. This is a contradiction, since $\varphi$ is not bounded by the argument in the case $d=n$ above. Thus such $t_{2} \in I$ exists.

We set

$$
\gamma=\frac{n}{n-1} \frac{d-1}{d}<1
$$

as above. We may assume that $t_{0} \in I \cap\left(t_{2}, \infty\right)$. 
Since (7.14) yields

$$
\varphi(t)^{1-\gamma} \geqslant \varphi\left(t_{0}\right)^{1-\gamma}+(1-\gamma) \beta\left(\psi_{n-1}\left(c_{D} t\right)-\psi_{n-1}\left(t_{0}\right)\right),
$$

we have that (a) holds with $\alpha=1 /(1-\gamma)$ in the case $d<n$.

Proof of Theorem 2. Theorem 2 follows immediately from Theorem 12. Indeed, if $\mathbb{X}=\mathbb{R}^{n}$ then there exists an exhaustion $\mathcal{D}=\left\{B^{n}(r): r \in I\right\}$ so that $|I \cap(0, R)| \geqslant \frac{1}{4} R$ from $R>1$ and

$$
\sup _{r \in(1, R)}\left(f_{B^{n}(r)} K^{n-1}\right)^{1 /(b-1)} \lesssim \mathfrak{K}_{n-1}(R) \lesssim \sup _{r \in(1, R)}\left(f_{B^{n}(r)} K^{n-1}\right)^{1 /(n-1)}
$$

whenever $R \in I$, see Remark 3. Combining this with (1.3) we find that $\mathfrak{K}_{n-1}(t)$ is bounded. Therefore, we have $\psi_{n-1}(t) \log t \gtrsim 1$ for all $t \in I \cap$ $\left(t_{\mathcal{D}}, \infty\right)$. Here the constant of comparability depends only on $n$.

Next we formulate a sharp version of Theorem 1.

Theorem 13. Let $\mathbb{X}$ be a complete, connected, and oriented Riemannian nmanifold and $\mathcal{D}=\left\{D_{t}: t \in I\right\}$ a controlled exhaustion of $\mathbb{X}$. Let $\left(\mathbb{Y}, g_{\mathbb{Y}}\right)$ be a closed, connected, and oriented Riemannian n-manifold and let $f: \mathbb{X} \rightarrow \mathbb{Y}$ be a non-constant mapping of finite distortion with distortion $K: \mathbb{X} \rightarrow[1, \infty)$. Let $p \in[n-1, n)$ and that either

(i) $p>n-1$ and $\operatorname{ker} f^{*} \neq 0$, or

(ii) $p=n-1, n \geqslant 4$, and $\operatorname{ker}\left\{f^{*}: H^{\ell}(\mathbb{Y}) \rightarrow H^{\ell}(\mathbb{X})\right\} \neq 0$ for some $\ell \in\{2, \ldots, n-2\}$.

If $\sup _{t \in I} \psi_{K, p}(t)=\infty$ then there exists $\alpha=\alpha\left(n, p, C_{g_{\mathbb{Y}}},[\mathcal{D}]\right)>0$ so that

$$
\liminf _{t \rightarrow \infty, t \in I} \frac{1}{e^{\alpha \psi_{p}\left(c_{D} t\right)}} \int_{D_{t}} J_{f}>0,
$$

where $c_{D}>0$ is as in $\left(D_{5}\right)$.

Proof. We may assume that $t_{\mathcal{D}} \geqslant 1$. Set $q=n p /(p+1)$. Then $n-1 \leqslant q<n$ and $1-n / q=1 / p$. Applying Theorem 10 with exponent $q$ and Hölder's inequality with $n / q$ and with $n /(n-1)$, we obtain, for $t \in I \cap\left(t_{\mathcal{D}}, \infty\right)$,

$$
\begin{aligned}
\int_{D_{t}} J_{f} & \leqslant C\left|\partial D_{t}\right|^{\frac{n}{n-1}-\frac{n}{q}}\left(\int_{\partial D_{t}}|D f|^{q}\right)^{\frac{n}{q}}+C \int_{\partial D_{t}}|D f|^{n-1} \\
& \leqslant C\left|\partial D_{t}\right|^{\frac{n}{n-1}-\frac{n}{q}}\left(\int_{\partial D_{t}} K^{p}\right)^{1 / p} \int_{\partial D_{t}} J_{f}+C\left(\int_{\partial D_{t}} K^{n-1}\right)^{1 / n} \int_{\partial D_{t}} J_{f} \\
& \leqslant C\left|\partial D_{t}\right|^{\frac{1}{n-1}} \mathfrak{K}_{p}(t) \int_{\partial D_{t}} J_{f}+C\left|\partial D_{t}\right|^{\frac{1}{n}} \mathfrak{K}_{n-1}(t)^{\frac{n-1}{n}} \int_{\partial D_{t}} J_{f} \\
& \leqslant C c_{A} t \mathfrak{K}_{p}(t) \int_{\partial D_{t}} J_{f},
\end{aligned}
$$


where $C$ is the constant in Theorem 10 . Here we also used the facts that $\left|\partial D_{t}\right|^{1 / n} \leqslant c_{A} t,\left|\partial D_{t}\right|^{1 /(n-1)} \leqslant c_{A} t$, and $\mathfrak{K}_{p}(t) \geqslant \mathfrak{K}_{n-1}(t) \geqslant 1$ for every $t \in I \cap\left(t_{\mathcal{D}}, \infty\right)$.

Let

$$
\varphi(t)=\int_{D_{t}} J_{f}
$$

for $t \in I$. Then

$$
\varphi^{\prime}(t) \geqslant c_{I} \int_{\partial D_{t}} J_{f}
$$

for almost every $t \in I$. Since $f$ is not constant, there exists $t_{0} \in I \cap\left(t_{\mathcal{D}}, \infty\right)$ so that $\varphi\left(t_{0}\right)>0$. Thus

$$
\begin{aligned}
\log \frac{\varphi(t)}{\varphi\left(t_{0}\right)} & \geqslant \int_{I \cap\left(t_{0}, t\right)} \frac{\varphi^{\prime}(s)}{\varphi(s)} \mathrm{d} s \geqslant \alpha \int_{I \cap\left(t_{0}, t\right)} \frac{\mathrm{d} s}{s \mathfrak{K}_{p}(s)} \\
& \geqslant \alpha \int_{t_{0}}^{t_{0}+\left|I \cap\left(t_{0}, t\right)\right|} \frac{\mathrm{d} s}{s \mathfrak{K}_{p}(s)} \\
& =\alpha\left(\psi\left(t_{0}+\left|I \cap\left(t_{0}, t\right)\right|\right)-\psi\left(t_{0}\right)\right) \\
& \geqslant \alpha\left(\psi\left(c_{D} t\right)-\psi\left(t_{0}\right)\right),
\end{aligned}
$$

where $\alpha$ depends on $n, p, C_{g_{Y}}$, and $[\mathcal{D}]$. Thus

$$
\frac{\varphi(t)}{e^{\alpha \psi\left(c_{D} t\right)}} \geqslant \varphi\left(t_{0}\right) e^{-\alpha \psi\left(t_{0}\right)}
$$

The claim follows.

Proof of Theorem 1. Since $f$ has mean distortion in $L^{p}$ we have $\psi_{p}(t) \sim \log t$ for $t \in I$ where $I$ is as in the proof of Theorem 2. Therefore, Theorem 1 follows from Theorem 13 with the exhaustion $\left\{B^{n}(r) ; r \in I\right\}$ and we have $\beta=\alpha / \hat{K}_{f, p}$.

Example 14. The following construction demonstrates the sharpness of the assumption $\sup _{t \in I} \psi_{K, p}(t)=\infty$ in Theorems 12 and 13. The construction is well-known in the context of locally quasiregular mappings and due to Zorich [23]; see also [11] for a Liouville type theorem in this context. We thank Peter Lindqvist for these references.

Suppose that we are given a measurable function $M:[1, \infty) \rightarrow[1, \infty)$ so that

$$
\int_{1}^{\infty} \frac{\mathrm{d} t}{t M(t)}<\infty
$$

Then first, we define $\tilde{M}(t)=M(t)$ for $t \geqslant 1$ and $\tilde{M}(t)=t^{-2}$ for $0<t<1$. Second, we set

$$
F(s)=\int_{0}^{s} \frac{\mathrm{d} t}{t \tilde{M}(t)} \quad \text { and } \quad \tilde{F}(s)=\frac{F(s)}{F(\infty)},
$$


where $F(\infty)=\lim _{s \rightarrow \infty} F(s)$. Now, we are ready to define a homeomorphism $f: \mathbb{R}^{n} \rightarrow B^{n}$ by the rule

$$
f(x)=\tilde{F}(|x|) \frac{x}{|x|}
$$

for all $x \in \mathbb{R}^{n}$. A simple computation shows that the mapping $f$ has finite distortion $K$ with

$$
\left(f_{\partial B_{t}} K^{p}\right)^{1 / p} \sim M(t)
$$

for almost every $t \geqslant 1$ and all $p \geqslant n-1$, where the constants of comparability depend only on $F(\infty)$. Together with the assumption (7.19) we have that $\sup _{r \geqslant 1} \psi_{p}(r)<\infty$. Since $B^{n}$ can be embedded into any manifold with a bilipschitz embedding, our construction is completed.

Both Theorems 12 and 13 admit local versions in the context of manifolds with boundary. We formulate a local version of Theorem 13 generalizing [15, Theorem 1].

Theorem 15. Let $\mathbb{X}$ be a complete, connected, and oriented Riemannian $n$-manifold with a compact boundary $\partial \mathbb{X}, \mathcal{D}=\left\{D_{t}: t \in I\right\}$ a controlled exhaustion of $\mathbb{X}$, and let $\left(\mathbb{Y}, g_{\mathbb{Y}}\right)$ be a closed, connected, and oriented Riemannian n-manifold. Suppose $f: \mathbb{X} \rightarrow \mathbb{Y}$ is a mapping of finite distortion with distortion $K: \mathbb{X} \rightarrow \mathbb{R}$. Let $p \in[n-1, n)$ and suppose that either

(i) $f$ has mean distortion in $L^{n-1}, n \geqslant 4$, and $\operatorname{ker}\left\{f^{*}: H^{\ell}(\mathbb{Y}) \rightarrow H^{\ell}(\mathbb{X})\right\} \neq$ 0 for some $\ell \in\{2, \ldots, n-2\}$, or

(ii) $f$ has mean distortion in $L^{p}$ for some $p>n-1$ and $\operatorname{ker} f^{*} \neq 0$. If $\sup _{t \in I} \psi_{K, p}(t)=\infty, J_{f} \mid \partial \mathbb{X} \in L^{1}(\partial \mathbb{X})$, and there exists $t_{0}>0$ such that

$$
\int_{D_{t_{0}}} J_{f} \geqslant 2 C c_{A} t_{0} \mathfrak{K}_{p, K_{f}}\left(t_{0}\right) \int_{\partial \mathbb{X}} J_{f} .
$$

where $C$ is the constant of Theorem 10, then there exists $\alpha=\alpha\left(n, p, C_{g_{Y}},[\mathcal{D}]\right)>$ 0 so that

$$
\liminf _{t \rightarrow \infty, t \in I} \frac{1}{e^{\alpha \psi\left(c_{D} t\right)}} \int_{D_{t}} J_{f}>0
$$

Proof. As the proof is merely a modification of the proof of Theorem 13, we only indicate the differences. Let

$$
\varphi(t)=\int_{D_{t}} J_{f}
$$

as in the proof of Theorem 13. Then

$$
\varphi^{\prime}(t) \geqslant c_{I} \int_{\partial D_{t} \backslash \partial \mathbb{X}} J_{f}
$$

for almost every $t \in I$. It is now sufficient to observe that by (7.17) and (7.20) we have

$$
\int_{D_{t}} J_{f} \leqslant C c_{A} t \mathfrak{K}_{p}(t) \int_{\partial D_{t}} J_{f} \leqslant C c_{A} t \mathfrak{K}_{p}(t) \int_{\partial D_{t} \backslash \partial \mathbb{X}} J_{f}+\frac{1}{2} \int_{D_{t_{0}}} J_{f}
$$


for $t \in I \cap\left(t_{0}, \infty\right)$. The claim now follows from (7.18).

\section{REFERENCES}

[1] M. Bonk and J. Heinonen. Quasiregular mappings and cohomology. Acta Math., 186(2):219-238, 2001.

[2] T. Coulhon, A. Grigor' yan, and D. Levin. On isoperimetric profiles of product spaces. Comm. Anal. Geom., 11(1):85-120, 2003.

[3] L. C. Evans and R. F. Gariepy. Measure theory and fine properties of functions. Studies in Advanced Mathematics. CRC Press, Boca Raton, FL, 1992.

[4] F. Giannetti and A. Passarelli di Napoli. Isoperimetric type inequalities for differential forms on manifolds. Indiana Univ. Math. J., 54(5):1483-1497, 2005.

[5] P. Hajłasz, T. Iwaniec, J. Malý, and J. Onninen. Weakly differentiable mappings between manifolds. Mem. Amer. Math. Soc., 192(899):viii+72, 2008.

[6] E. Hebey. Sobolev spaces on Riemannian manifolds, volume 1635 of Lecture Notes in Mathematics. Springer-Verlag, Berlin, 1996.

[7] J. Heinonen. Lectures on analysis on metric spaces. Universitext. Springer-Verlag, New York, 2001.

[8] I. Holopainen and S. Rickman. Classification of Riemannian manifolds in nonlinear potential theory. Potential Anal., 2(1):37-66, 1993.

[9] T. Iwaniec and G. Martin. Geometric function theory and non-linear analysis. Oxford Mathematical Monographs. The Clarendon Press Oxford University Press, New York, 2001.

[10] T. Iwaniec, C. Scott, and B. Stroffolini. Nonlinear Hodge theory on manifolds with boundary. Ann. Mat. Pura Appl. (4), 177:37-115, 1999.

[11] P. Lindqvist. On Liouville's theorem for locally quasiregular mappings in $\mathbf{R}^{n}$. Israel J. Math., 47(4):291-295, 1984.

[12] J. J. Manfredi and E. Villamor. Mappings with integrable dilatation in higher dimensions. Bull. Amer. Math. Soc. (N.S.), 32(2):235-240, 1995.

[13] P. Mattila and S. Rickman. Averages of the counting function of a quasiregular mapping. Acta Math., 143(3-4):273-305, 1979.

[14] M. Miranda, Jr., D. Pallara, F. Paronetto, and M. Preunkert. Heat semigroup and functions of bounded variation on Riemannian manifolds. J. Reine Angew. Math., 613:99-119, 2007.

[15] P. Pankka. Slow quasiregular mappings and universal coverings. Duke Math. Jour., 141(2):293-320, 2008.

[16] S. Rickman. Quasiregular mappings, volume 26 of Ergebnisse der Mathematik und ihrer Grenzgebiete (3) [Results in Mathematics and Related Areas (3)]. SpringerVerlag, Berlin, 1993.

[17] R. Schoen and K. Uhlenbeck. A regularity theory for harmonic maps. J. Differential Geom., 17(2):307-335, 1982.

[18] R. Schoen and K. Uhlenbeck. Boundary regularity and the Dirichlet problem for harmonic maps. J. Differential Geom., 18(2):253-268, 1983.

[19] C. Scott. $L^{p}$ theory of differential forms on manifolds. Trans. Amer. Math. Soc., 347(6):2075-2096, 1995.

[20] N. T. Varopoulos, L. Saloff-Coste, and T. Coulhon. Analysis and geometry on groups, volume 100 of Cambridge Tracts in Mathematics. Cambridge University Press, Cambridge, 1992.

[21] F. W. Warner. Foundations of differentiable manifolds and Lie groups, volume 94 of Graduate Texts in Mathematics. Springer-Verlag, New York, 1983.

[22] W. P. Ziemer. Extremal length and conformal capacity. Trans. Amer. Math. Soc., 126:460-473, 1967. 
[23] V. A. Zorich. Admissible order of growth of the quasiconformality characteristic in lavrentiev's theorem. Dokl. Akad. Nauk. SSSR, 181:866-869, 1968.

[24] V. A. Zorich. Asymptotics of the admissible growth of the coefficient of quasiconformality at infinity and injectivity of immersions of Riemannian manifolds. Publ. Inst. Math. (Beograd) (N.S.), 75(89):53-57, 2004.

J.O. Syracuse University, Department of Mathematics, 215 Carnegie Hall, Syracuse, NY 13244-1150, USA. e-mail: jkonnine@syr.edu

P.P. University of Michigan, Department of Mathematics, 530 Church Street, Ann Arbor, MI 48109-0937, USA. e-mail: pankka@umich.edu 